\title{
Influence of training status on high-intensity intermittent performance in response to $\beta$-alanine supplementation
}

\author{
Vitor de Salles Painelli • Bryan Saunders $\cdot$ Craig Sale $\cdot$ \\ Roger Charles Harris • Marina Yázigi Solis • Hamilton Roschel • \\ Bruno Gualano - Guilherme Giannini Artioli • Antonio Herbert Lancha Jr.
}

Received: 14 August 2013/Accepted: 17 January 2014/Published online: 6 February 2014

(C) The Author(s) 2014. This article is published with open access at Springerlink.com

\begin{abstract}
Recent investigations have suggested that highly trained athletes may be less responsive to the ergogenic effects of $\beta$-alanine (BA) supplementation than recreationally active individuals due to their elevated muscle buffering capacity. We investigated whether training status influences the effect of BA on repeated Wingate performance. Forty young males were divided into two groups according to their training status (trained: $\mathrm{T}$, and non-trained: NT cyclists) and were randomly allocated to $\mathrm{BA}$ and a dextrose-based placebo (PL) groups, providing four experimental conditions: NTPL, NTBA, TPL, TBA. BA $\left(6.4 \mathrm{~g} \mathrm{day}^{-1}\right)$ or PL was ingested for 4 weeks, with participants completing four 30 -s lower-body Wingate bouts, separated by $3 \mathrm{~min}$, before and after supplementation. Total work done was significantly increased following supplementation in both NTBA $(p=0.03)$ and TBA $(p=0.002)$, and it was significantly reduced in NTPL $(p=0.03)$ with no difference for TPL $(p=0.73)$. BA supplementation increased mean power output (MPO) in bout 4 for the NTBA group ( $p=0.0004)$ and in bouts 1,2 and 4 for the TBA group $(p \leq 0.05)$. No differences were observed in MPO for NTPL and TPL. BA supplementation was effective at improving repeated high-intensity cycling
\end{abstract}

V. de Salles Painelli - B. Saunders - M. Y. Solis - H. Roschel · B. Gualano · G. G. Artioli ( $\varangle)$ · A. H. Lancha Jr. Laboratory of Applied Nutrition and Metabolism, School of Physical Education and Sport, University of Sao Paulo, Av. Mello de Moraes, 65-Butanta, São Paulo, SP 05508-030, Brazil e-mail: artioli@usp.br

B. Saunders - C. Sale

Biomedical, Life and Health Sciences Research Centre,

Nottingham Trent University, Nottingham NG11 8NS, UK

R. C. Harris

Junipa Ltd, Newmarket, Suffolk, UK performance in both trained and non-trained individuals, highlighting the efficacy of BA as an ergogenic aid for high-intensity exercise regardless of the training status of the individual.

Keywords Carnosine $\cdot$ Buffers $\cdot$ Athletes $\cdot$ Dietary supplement $\cdot \beta$-Alanine $\cdot$ Performance

\section{Introduction}

Carnosine ( $\beta$-alanyl-L-histidine) is a cytoplasmic dipeptide found in high concentrations in the skeletal muscle of vertebrates and non-vertebrates, as well as in the central nervous system. Carnosine may play multiple roles in muscle, such as antioxidant (Boldyrev et al. 1993) and antiglycant (Hipkiss et al. 1995). In addition, recent studies have shown that increased carnosine content in muscle fibres may improve the $\mathrm{Ca}^{2+}$ sensitivity of the contractile apparatus and enhance $\mathrm{Ca}^{2+}$ release from sarcoplasmic reticulum when $\mathrm{Ca}^{2+}$ release is inhibited, such as during fatigue (Dutka et al. 2012). However, it is undisputed that intramuscular $\mathrm{pH}$ buffering is a major function of carnosine, a consequence of the $\mathrm{pKa}$ of the imidazole ring of carnosine being 6.83 (Bate-Smith 1938).

Harris et al. (2006) showed that $\beta$-alanine (BA) availability is the rate-limiting factor for carnosine synthesis in skeletal muscle and that $\beta$-alanine supplementation is able to increase the intramuscular content of carnosine. After this seminal study by Harris and colleagues, several investigations have examined the ergogenic effects of $\beta$ alanine supplementation on exercise performance and capacity [for a review, see Sale et al. (2013)] and a recent meta-analysis concluded that supplementation significantly improves exercise capacity, but not sport-related 
performance (Hobson et al. 2012). The body of evidence surrounding the ergogenic effects of $\beta$-alanine supplementation is increasing; however, the majority of studies that have shown a positive effect have used recreationally active participants. Although some studies have shown positive effects of $\beta$-alanine on trained athletes (Tobias et al. 2013; Salles Painelli et al. 2013; Van Thienen et al. 2009; Derave et al. 2007), several investigations have shown no improvements or marginal effects (Derave et al. 2007; Baguet et al. 2010; Saunders et al. 2012; Hobson et al. 2013; Ducker et al. 2013; Chung et al. 2012; Howe et al. 2014). As such, the ergogenic effects of $\beta$-alanine in athletes are less clear than in non-trained individuals. In addition, some authors speculate that athletes might be less responsive to $\beta$-alanine supplementation (Bellinger et al. 2012; Bellinger 2014).

It has been shown that trained athletes have an elevated muscle buffering capacity as an adaptation to exercise training (Weston et al. 1997; Edge et al. 2006a, b). Since most studies that recruited athletes have failed to demonstrate an ergogenic effect of $\beta$-alanine, it has been speculated that this may be due to the already increased buffering capacity in highly trained individuals, limiting the measurable effect of increased carnosine content through $\beta$-alanine supplementation (Bellinger et al. 2012; Bellinger 2014). Although athletic populations are likely to be the most interested in the ergogenic effects of $\beta$-alanine, it remains unclear to what extent these highly trained individuals can benefit from supplementation. No study to date has directly compared whether athletes respond differently to $\beta$-alanine supplementation in comparison to non-trained recreationally active individuals.

Therefore, to gather knowledge on the potential differential responses to $\beta$-alanine supplementation between trained and non-trained participants, the aim of this study was to investigate whether training status influences the ergogenic effect of $\beta$-alanine supplementation on repeated Wingate performance using both trained and non-trained cyclists.

\section{Methods}

\section{Participants}

Forty young male individuals participated in this study. They were allocated to two groups according to their training status (endurance-trained cyclists: $N=20$; nontrained individuals: $N=20$ ). Participants were further randomly allocated to the $\beta$-alanine and placebo (PL) groups, matched for total work done (TWD) measured during the habituation session. One trained cyclist withdrew from the study due to injury, meaning that 19 trained cyclists were included in the final data set. The cyclists were all actively involved in structured training programs and, although endurance training formed a large part of their training routine, all athletes also undertook regular sprint training. Thirteen of these athletes were competing at national level and the remaining six athletes were participating in state-level official competitions at the time of data collection. The non-trained participants were recreationally active individuals who engaged in a variety of activities (e.g., weightlifting, running, team sports) 1-3 times per week. Participant characteristics, experience and weekly training volumes at the moment they initiated the study are presented in Table 1 . The ability to perform high-intensity intermittent exercise was clearly different between the trained and the non-trained groups (Table 2).

Participants were required not to have taken any creatine supplement for 3 months prior to the study and had not taken $\beta$-alanine for at least 6 months prior to the study. Participants were also requested to maintain similar levels of physical activity and dietary intake for the duration of the study and compliance with this request was verbally confirmed with participants prior to commencement of the study. Participants were fully informed of any risks and discomforts associated with the study before completing a health screen and providing written consent. The study was approved by the Ethical Advisory Committee from the School of Physical Education and Sport, University of Sao Paulo (Approval Number: 2011/15).

Table 1 Participant characteristics

\begin{tabular}{lllcc}
\hline & NTPL $(N=10)$ & NTBA $(N=10)$ & TPL $(N=9)$ & TBA $(N=10)$ \\
\hline Age (years) & $26 \pm 4$ & $25 \pm 4$ & $33 \pm 12$ & $32 \pm 8$ \\
Body mass $(\mathrm{kg})$ & $72.6 \pm 8.8$ & $77.6 \pm 9.9$ & $68.9 \pm 10.0$ & $71.7 \pm 5.5$ \\
Height $(\mathrm{m})$ & $1.75 \pm 0.08$ & $1.80 \pm 0.07$ & $1.79 \pm 0.07$ & $1.82 \pm 0.05$ \\
Weekly training volume $(\mathrm{km})$ & - & - & $230 \pm 165$ & $278 \pm 94$ \\
Training experience (years) & - & - & $9 \pm 6$ & $8 \pm 8$ \\
\hline
\end{tabular}

NTPL non-trained + PL, NTBA non-trained + BA, TPL trained + PL, TBA trained + BA. No differences were observed between groups 
Table 2 Performance in the four bouts of the Wingate test according to training status

\begin{tabular}{lclc}
\hline & $\begin{array}{l}\text { Non-trained } \\
(N=20)\end{array}$ & $\begin{array}{l}\text { Trained } \\
(N=19)\end{array}$ & $P$ \\
\hline TWD $(\mathrm{J})$ & $49,093 \pm 6,043$ & $54,399 \pm 6,603$ & 0.013 \\
Performance & $32.66 \pm 7.38$ & $11.93 \pm 6.39$ & $<0.0001$ \\
decrement $(\%)^{\mathrm{a}}$ & & & \\
Relative peak power output & & & \\
1st bout (W/kg) & $8.18 \pm 0.70$ & $8.16 \pm 0.77$ & 0.940 \\
2nd bout (W/kg) & $7.73 \pm 0.59$ & $8.03 \pm 0.80$ & 0.196 \\
3rd bout (W/kg) & $6.89 \pm 0.71$ & $7.79 \pm 0.69$ & $<0.0001$ \\
4th bout (W/kg) & $5.99 \pm 0.87$ & $7.50 \pm 0.66$ & $<0.0001$ \\
Relative mean power output & & \\
1st bout (W/kg) & $6.67 \pm 0.46$ & $6.86 \pm 0.51$ & 0.238 \\
2nd bout (W/kg) & $5.74 \pm 0.42$ & $6.54 \pm 0.52$ & $<0.0001$ \\
3rd bout (W/kg) & $4.93 \pm 0.52$ & $6.29 \pm 0.50$ & $<0.0001$ \\
4th bout $(\mathrm{W} / \mathrm{kg})$ & $4.48 \pm 0.53$ & $6.04 \pm 0.51$ & $<0.0001$ \\
\hline
\end{tabular}

${ }^{\text {a }}$ Decrement in work done from the 1 st bout to the 4 th bout

\section{Experimental design}

Participants attended the laboratory on three separate occasions. The first visit was for protocol habituation, with the remaining two visits for the completion of the main trials. One main trial was completed before and one main trial following a 4 week double-blind supplementation period of either $\beta$-alanine or placebo. As such, the study comprised four experimental conditions: nontrained + placebo (NTPL, $N=10)$, non-trained $+\beta$-alanine (NTBA, $N=10$ ), trained + placebo $(\mathrm{TPL}, N=9$ ) and trained $+\beta$-alanine (TBA, $N=10$ ).

Participants were supplemented for 4 weeks with either $6.4 \mathrm{~g} \mathrm{day}^{-1}$ BA (CarnoSyn $^{\mathrm{TM}}$, Compound Solutions Inc. $^{\mathrm{TM}}$, CA, USA) or an equivalent amount of placebo capsules (dextrose; Ethika Inc. ${ }^{\text {TM }}$, São Paulo, Brazil). This dosing regimen has been proven to be effective in increasing muscle carnosine content over 2 (Stellingwerff et al. 2012) and 4 weeks (Harris et al. 2006), and it is $\sim 10-20$ times higher than the average $\beta$-alanine intake from food, as calculated for the participants of the present study (results are shown in Table 3). The participants took two $800-\mathrm{mg}$ gelatin capsules four times per day at 3-4 h intervals. Carboxymethyl cellulose was added to the $\beta$ alanine capsules (100 mg per $800 \mathrm{mg}$ of $\beta$-alanine) in order to slow the absorption of $\beta$-alanine and minimize paraesthesia. Participants received exactly 4 weeks' worth of supplement in a sealed, unmarked container, and compliance was monitored by verifying the remaining contents of the container upon return to the lab following the supplementation period. The degree of compliance was reported to be $100 \%$ in all groups.

\section{Experimental procedures}

\section{Preliminary testing}

Height and body mass were recorded upon arrival at the laboratory. The exercise protocol required participants to complete 4 bouts of a modified lower-body cycling Wingate test (Inbar and Bar-Or 1986), using a specifically designed ergometer (Biotec 2100, Cefise, Brazil). The position on the cycle ergometer was determined prior to the habituation session, recorded and maintained for all subsequent trials. The participants' feet were securely attached to the pedals using toe clips and straps. Participants warmed up for $5 \mathrm{~min}$ on the ergometer prior to the first bout, against no resistance. On completion of the warm up, the test began immediately from a static start. Each Wingate bout lasted $30 \mathrm{~s}$ and participants were required to cycle against a load set at $5 \%$ of body mass, which was measured with a digital scale to the nearest $10 \mathrm{~g}$. This $5 \%$ load was chosen over the original $7.5 \%$ load in order to allow the non-trained participants to complete the entire 4-bout protocol. Bouts were interspersed by 3 -min passive recovery, with all the participants being seated during this recovery period. Standardised verbal encouragement was given throughout every bout. Upon completion of the test, the resistance was removed and the participants were instructed to continue cycling at a self-selected cadence to facilitate recovery. A set of 24 sensors measured wheel velocity, with power output being calculated automatically every second by computer software (Ergometric 6.0, Cefise, Brazil). Mean power (MPO, W) and peak power (PPO, W) output were obtained for each bout and TWD (J) was obtained for the overall test session. Performance decrement was measured as the percentage loss in work done from the 1st to the 4th bout. The coefficient of variation for TWD in the non-trained and the trained groups was $2.42 \pm 1.86$ and $1.78 \pm 1.27 \%$, respectively.
Table 3 Energy, macronutrient and $\beta$-alanine intake

\begin{tabular}{lcccc}
\hline & NTPL $(N=10)$ & NTBA $(N=10)$ & TPL $(N=9)$ & TBA $(N=10)$ \\
\hline Energy (Kcal) & $2,747 \pm 661$ & $3,364 \pm 662$ & $3,181 \pm 430$ & $2,511 \pm 769$ \\
Carbohydrate $(\mathrm{g})$ & $342 \pm 96$ & $448 \pm 86$ & $364 \pm 50$ & $304 \pm 64$ \\
Protein $(\mathrm{g})$ & $134 \pm 37$ & $150 \pm 39$ & $140 \pm 42$ & $111 \pm 39$ \\
Fat $(\mathrm{g})$ & $94 \pm 35$ & $108 \pm 47$ & $129 \pm 23$ & $95 \pm 52$ \\
$\beta$-Alanine $(\mathrm{mg})$ & $383 \pm 191$ & $397 \pm 192$ & $446 \pm 328$ & $364 \pm 283$ \\
\hline
\end{tabular}




\section{Main trials}

Participants were requested to abstain from alcohol and strenuous exercise in the $24-\mathrm{h}$ period prior to the main trials. Participants arrived at the laboratory in at least $2 \mathrm{~h}$ following their last meal, and immediately began their warm up followed by the 4-bout lower-body cycling Wingate test. Ad libitum water consumption was allowed during the main trials. To control for intervening variables, food intake was assessed during the supplementation period by means of three $24-\mathrm{h}$ dietary recalls undertaken on separate days ( 2 weekdays and 1 weekend day), with the aid of a visual photo album of real-sized foods and portions. Nutritional supplements were also recorded. Energy and macronutrient intakes were analysed by the software Virtual Nutri $^{\text {TM }}$ (São Paulo, Brazil). The intake of $\beta$-alanine from fish and red meats containing carnosine and its related derivatives was estimated from the data of Jones et al. (2011).

\section{Statistical analyses}

All data are presented as mean $\pm \mathrm{SD}$ and were analysed using the SAS statistical package, (SAS version 9.2). A one-way ANOVA, with Group (i.e. NTPL, NTBA, TPL and TBA) as a fixed factor, was used to compare the participant characteristics and baseline performance between groups. The mixed models for repeated measures were used to examine the effect of supplementation on TWD, with Group and Time (i.e. pre-supplementation, post-supplementation) as fixed factors. To mitigate the impact of inter-individual data variability, all TWD values were converted into delta scores (i.e., POST-PRE values) and thereafter tested by a mixed model assuming "pre values" as a covariate. The mixed models were also performed to examine the effect of supplementation on MPO and PPO, with Group, Time and Bout (i.e. bout 1, bout 2, bout 3, bout 4) as fixed factors. Tukey tests were used for post hoc analyses and effect sizes were calculated using Cohen's d. Statistical significance was accepted at $p \leq 0.05$, with a trend towards significance being accepted at $p \leq 0.1$.

\section{Results}

Wingate test

\section{Effects of $\beta$-alanine supplementation regardless of training status}

There was no difference in TWD between placebo and $\beta$ alanine groups prior to supplementation (placebo: $50,773 \pm 7,349 \mathrm{~J} ; \quad \beta$-alanine: $\quad 52,538 \pm 6,283 \mathrm{~J}$;

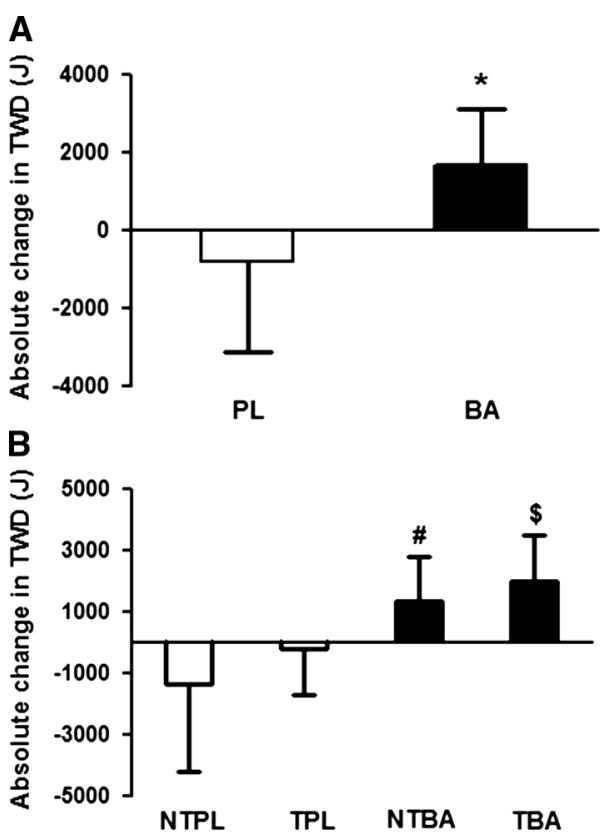

Fig. 1 a Absolute change in performance in placebo $(P L)$ and $\beta$ alanine $(B A)$ with trained and non-trained participants grouped together. ${ }^{*} p \leq 0.001$ from placebo. b Absolute change in performance for individual groups NTPL, TPL, NTBA and TBA. ${ }^{\#} p=0.008$ from NTPL. ${ }^{\$} p=0.037$ from TPL. Effect sizes: NTPL -0.2 ; TPL -0.03 ; NTBA-0.2; TBA -0.4

$p=0.44)$. There was a significant interaction effect of Group $\times$ Time on TWD $(F=16.48 ; p=0.0002)$; following supplementation, TWD was increased by $1,663 \pm 1,457 \mathrm{~J}$ in the $\beta$-alanine group $(p=0.0004)$ and decreased by $832 \pm 2,308 \mathrm{~J}$ in the placebo group ( $p=0.07$; Fig. 1a). A total of 17 out of the 20 participants supplemented with $\beta$-alanine improved TWD following supplementation; 8 out of the 10 participants in the NTBA improved their performance, while 9 out of the 10 in the TBA group showed improved performance (Fig. 2a). On the other hand, only 10 out of the 19 participants supplemented with placebo improved TWD following supplementation; 5 out of the 10 participants in the NTPL improved their performance, while 5 out of the 9 in the TPL group showed improved performance (Fig. 2b).

Effects of $\beta$-alanine supplementation in trained versus nontrained subjects

Prior to supplementation, performance decrement from the $1 \mathrm{st}$ to the 4th Wingate bout was $32.3 \pm 6.6$ and $33.1 \pm 8.5 \%$ for the NTPL and the NTBA groups, respectively, with no differences between these two groups $(p>0.05)$. For the trained athletes, prior to the supplementation period, the performance decrement in the Wingate was $11.6 \pm 7.9$ and $12.3 \pm 5.0 \%$ for the TPL and the TBA, respectively $(p>0.05)$. Performance decrement for 

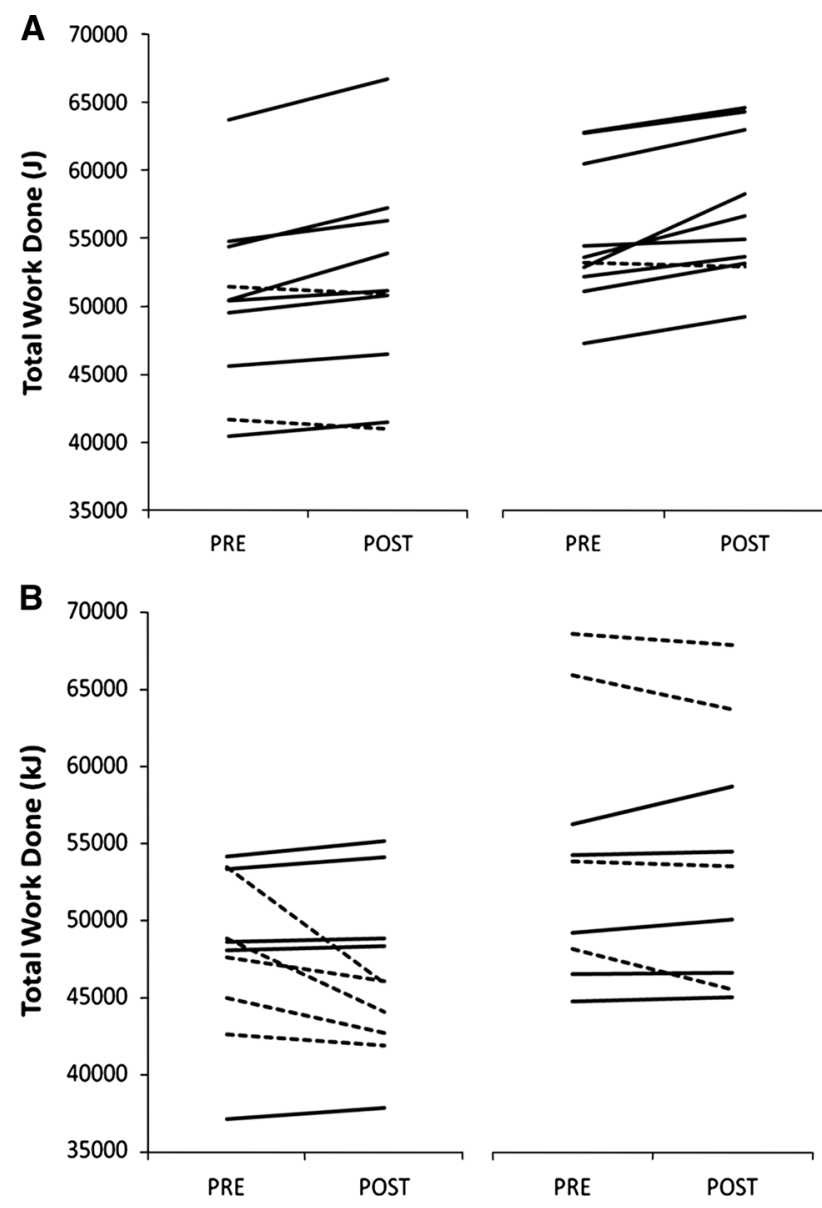

Fig. 2 a Individual response in TWD to supplementation in the $\beta$ alanine groups (NTBA and TBA, respectively). b Individual response in TWD to supplementation in the placebo groups (NTPL and TPL, respectively). Individuals who improved performance are indicated by a solid line, while those who did not are indicated by a dotted line

the NTPL and the NTBA groups was significantly higher when compared with both the TPL and the TBA groups (all $p<0.05)$.

TWD did not differ within the non-trained (NTPL: $47,913 \pm 5,313 \mathrm{~J}$ and NTBA: $50,274 \pm 6,765 \mathrm{~J}$; $p=0.43$ ) and trained groups (TPL: $53,951 \pm 8,258 \mathrm{~J}$ and TBA: $54,802 \pm 5,119 \mathrm{~J} ; p=0.78$ ) prior to supplementation. TWD was significantly increased following supplementation in both the NTBA $(+1,349 \pm 1,411 \mathrm{~J}$; $p=0.03) \quad$ and TBA groups $(+1,978 \pm 1,508 \mathrm{~J}$; $p=0.002)$, and it was significantly reduced in the NTPL $(-1,385 \pm 2,815 \mathrm{~J} ; p=0.03)$ with no significant difference being shown in the TPL group $(-219 \pm 1,507 \mathrm{~J}$; $p=0.73$ ). Analysis of covariance revealed that absolute change in TWD in both supplemented groups was significantly higher than in their respective placebo groups (NTBA vs. NTPL: $p=0.008$; TBA vs. TPL: $p=0.037$ ). However, no significant differences were shown between groups (NTPL vs. TPL: $p=0.10$; NTBA vs. TBA: $p=0.60)$ indicating that the effect of $\beta$-alanine supplementation was not affected by training status (Fig. 1b).

MPO was reduced in each subsequent bout during the pre-supplementation trial for all of the groups (Fig. 3). There was an interaction effect on MPO (Group $\times$ Time $\times$ Bout, $F=4.89 ; \quad p<0.0001) ;$ post hoc tests revealed that both supplemented groups (i.e. NTBA and TBA) were able to maintain MPO from bouts 3-4 following supplementation (both $p>0.05$ ). Compared to the pre-supplementation trial, post-supplementation MPO was higher in bout 4 for the NTBA $(p=0.0004)$, and higher in bouts 1,2 and 4 for the TBA group ( $p \leq 0.05$, Fig. 3).

In the pre-supplementation test, both of the non-trained groups (i.e. NTPL and NTBA) showed a significant reduction in PPO in every subsequent trial (all $p<0.05$ ). Conversely, both trained groups were able to maintain PPO across bouts pre-supplementation (all $p>0.05$ ). Importantly, there was an interaction effect on PPO (Group $\times$ Time $\times$ Bout, $F=2.70 ; \quad p=0.003)$, with increased values shown post-supplementation in bout 4 for the NTBA $(p=0.004)$; and a tendency for increased values in bout 2 for the TBA group $(p=0.08)$.

\section{Food intake}

The energy, carbohydrate, protein, fat and $\beta$-alanine intake are presented in Table 3. No significant differences were observed within or between groups (all $p>0.05$ ).

Blinding efficacy and side effects

Of the 20 participants who were supplemented with $\beta$ alanine, only 4 were able to correctly guess their supplement. Of the 19 participants who were supplemented with dextrose, only 9 were able to correctly guess their supplement (Fischer exact test: $p=0.33$ ). Three participants taking $\beta$-alanine reported mild paraesthesia and were able to correctly identify their supplement. We reanalysed the data without these three participants and the results remained similar, with no significant differences in the overall findings (as such, these data are not shown). No side effects related to dextrose ingestion were reported.

\section{Discussion}

Since there is the possibility that an already increased muscle buffering capacity explains the lack of a measurable ergogenic effect of $\beta$-alanine in trained athletes, we chose, for the first time, to directly investigate whether training status influences the ergogenic effect of $\beta$-alanine supplementation. The main findings of this study were that $\beta$-alanine supplementation improved repeated-bout high- 
Fig. 3 MPO during each bout of the Wingate test for NTPL (a), NTBA (b), TPL (c), and TBA (d). Pre- and postsupplementation MPO are indicated by white and black bars, respectively. ${ }^{*} p \leq 0.05$ from the previous bout. \# $p \leq 0.05$ from presupplementation during the same bout
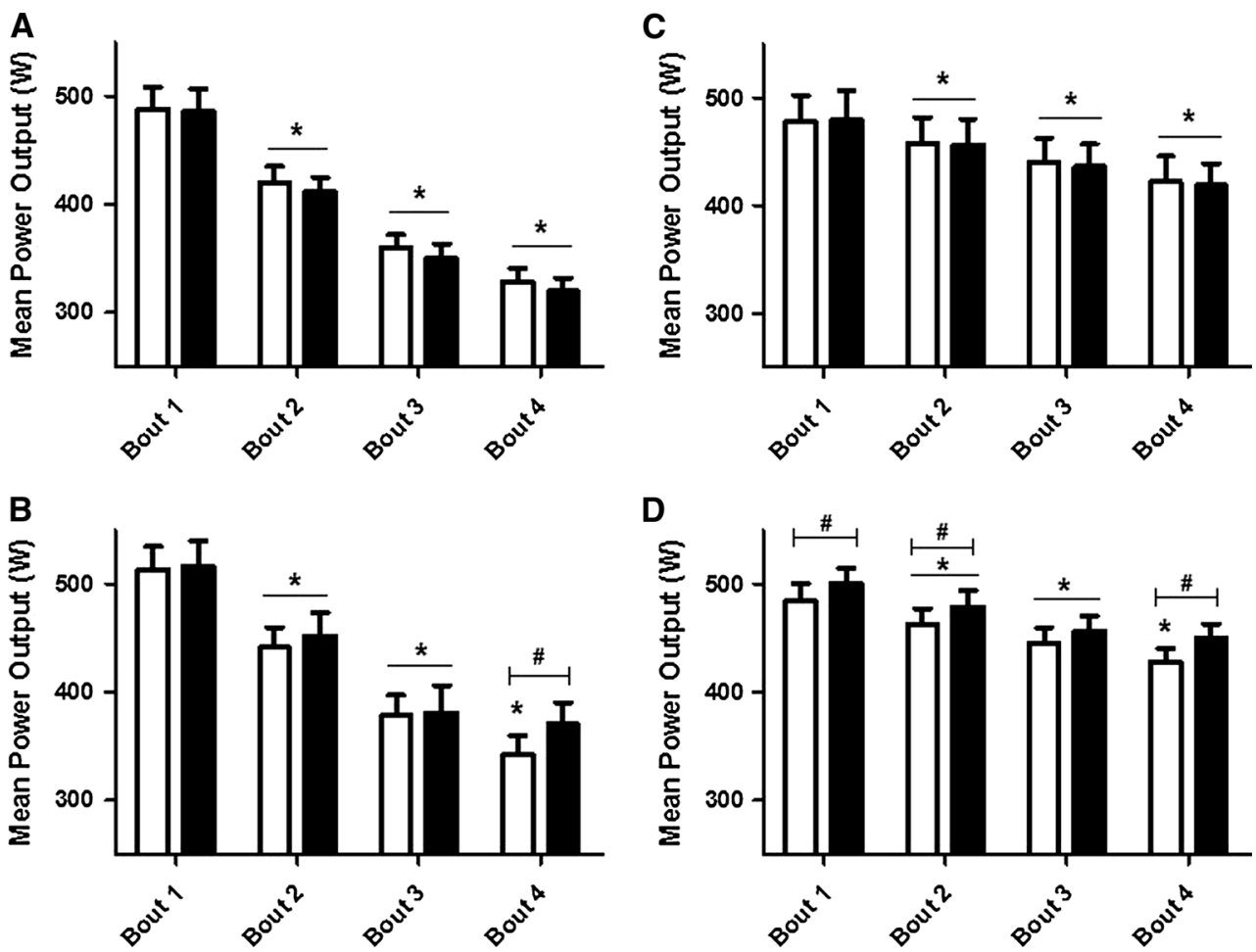

intensity cycling performance, to a similar extent, in both trained and non-trained participants.

The findings of our study are in contrast to previous studies that have shown no effect of $\beta$-alanine on highly trained individuals (Derave et al. 2007; Baguet et al. 2010; Bellinger et al. 2012; Saunders et al. 2012). The differences in results might be due to the protocols employed; previous studies may not have used exercise tests of sufficient intensity or duration to be influenced by decreased intramuscular $\mathrm{pH}$, or may have been susceptible to pacing strategies, masking any ergogenic effects of increased muscle buffering capacity. Intermittent supra-maximal exercise promotes a considerably greater intramuscular acidosis than continuous high-intensity exercise (Hermansen and Osnes 1972; Belfry et al. 2012), probably because the former is more reliant on glycolytic ATP resynthesis (Belfry et al. 2012). Thus, it is reasonable to assume that intermittent exercise performance is more likely to be limited by muscle acidosis and, hence, more susceptible to improvements with increased buffering capacity. The 4-bout lower-body modified Wingate test is extremely high-intensity in nature and requires the individual to perform maximally in every bout. Artioli et al. (2007) and De Salles Painelli et al. (2014) showed blood lactate values up to $\sim 15 \mathrm{mmol} \mathrm{L}^{-1}$ following the 4-bout upper-body Wingate test and venous blood $\mathrm{pH}$ as low as $\sim 7.05$ following 3 bouts of the upper-body Wingate test, highlighting the relevant contribution of anaerobic glycolysis for tests similar in duration, number of bouts and recovery between bouts. Therefore, the repeated-bout Wingate test would appear to be an appropriate model to detect performance improvements promoted by nutritional strategies that increase buffering capacity.

In the present study, TWD did not differ within the nontrained (NTPL vs. NTBA) and trained groups (TPL vs. TBA) prior to supplementation, indicating that randomisation successfully generated similar groups in each profile. Moreover, TWD prior to supplementation in the trained participants was $\sim 11 \%$ higher $(p<0.05)$ than in the nontrained participants, and work decrement from the 1 st to the 4th bout in the non-trained participants was $20 \%$ higher than in their trained counterparts (average 32 vs. $12 \%$ decrement, respectively; $p<0.05$ ). Since it has been demonstrated that the buffering capacity of homogenates of muscle (which is assumed to be a measure of muscle buffering capacity in situ) is related to repeated sprint ability and work decrement over repeated sprints (Bishop et al. 2004), it is plausible to assume that individuals in the trained groups have higher muscle buffering capacity than those in the non-trained groups. This, however, was not directly measured in the present investigation because it would require muscle biopsies, which are excessively invasive to be performed in athletes in the middle of the competitive season.

It should be noted that the peak and mean power attained in the 1st bout were not different between trained and non-trained. However, this was most likely caused by the lowered mechanical load of the Wingate test $(5 \%$ in 
the present study instead of the original $7.5 \%$ ) employed in our protocol, which hampered the Wingate test to distinguish the trained from the non-trained participants. On the other hand, this modification was necessary to allow the untrained individuals to complete the entire 4-bout protocol, which would not be possible for most of them if we used the $7.5 \%$ load. In spite of the lack of differences in the first bout, our 4-bout modified Wingate test was able to clearly differentiate both groups in terms of mean and peak power in the last $2-3$ bouts, TWD in the overall protocol and performance decrement over the 4 bouts.

Despite the remarkable differences in repeated sprint ability between the trained and non-trained individuals, four weeks of $\beta$-alanine supplementation increased TWD to a similar extent in both groups (non-trained: $2.52 \pm 2.64 \%$, trained: $3.64 \pm 2.87 \%$ ). The magnitude of the improvement in performance observed in the present study is in agreement with a recent meta-analysis that showed a $2.85 \%$ positive effect of $\beta$-alanine (Hobson et al. 2012). Although improvements in TWD around $3.0 \%$ may suggest only a discrete effect, it is worth noting that TWD in the trained participants was $\sim 11 \%$ higher than in their non-trained counterpart, meaning that 4 weeks of supplementation elicited a performance improvement which represents about onethird of the improvement achieved by several years of training.

In the present study, TWD increased due to an increased ability to maintain MPO during the Wingate bouts. A beneficial effect can be expected in the latter bouts of the test, since a single 30 -s maximal effort is unlikely to be affected by reduced intramuscular $\mathrm{pH}$ (Bogdanis et al. 1998). Artioli et al. (2007) employed an upper-body Wingate protocol to determine the effect of increased extracellular buffering and showed an increase in MPO in the last bout of exercise when experienced judo athletes were supplemented with sodium bicarbonate. Similarly, the non-trained participants supplemented with $\beta$-alanine in this study improved their MPO in the final bout of exercise, likely due to an increase in muscle buffering capacity over the exercise $\mathrm{pH}$ transit range, from increased carnosine concentrations. However, the trained participants supplemented with $\beta$-alanine showed an improved MPO across almost all of the bouts. This may be due to the training status of the trained athletes, who are likely to have had an increased ability to consistently perform maximal exercise across each bout, resulting in an earlier intramuscular $\mathrm{H}^{+}$ accumulation, placing an earlier reliance on their buffering capacity. Furthermore, it is possible that $\beta$-alanine supplementation provided a higher training quality during the supplementation period, enhancing training volume and/or intensity, thus increasing MPO in almost all of the bouts in TBA.
A potential limitation of this study is that we were unable to perform muscle analyses to confirm the efficacy of $\beta$-alanine in increasing muscle carnosine content. However, all human studies using 1.6-6.4 $\mathrm{g} \mathrm{day}^{-1}$ of $\beta$ alanine for 4 weeks or longer have so far reported increases of at least $8 \mathrm{mmol} \mathrm{kg}^{-1}$ dry muscle (corresponding to an increase of $40 \%$ in muscle carnosine) (Sale et al. 2013). According to the estimates by Harris et al. (2006), a $40 \%$ increase in muscle carnosine would represent a $\sim 4 \%$ increase in whole muscle buffering capacity and a $\sim 5 \%$ in type II fibres buffering capacity. Considering that all human studies with $\beta$-alanine supplementation have shown increased muscle carnosine, it appears to be safe to assume that our $\beta$-alanine supplementation protocol resulted in increased muscle carnosine. Also, based on the current findings showing that trained and non-trained participants equally improved their performance, it is reasonable to assume that carnosine accrual after $\beta$-alanine supplementation is not influenced by training status and training history. This is in agreement with Kendrick et al. (2009) using the same supplementation protocol as used in the present study and where the carnosine content of types I and II muscle fibres was measured directly, but in contrast with a recent study by Bex et al. (2014). The latter study, based on MRS measurements, suggested that carnosine elevation induced by $\beta$-alanine is higher in trained as compared to non-trained muscles. Further studies directly comparing the responses of both trained and non-trained individuals to $\beta$-alanine supplementation and its effects on performance and muscle carnosine content in different muscle fibre types are needed to clarify this issue.

It is important to note that 3 out of 20 of the participants ingesting $\beta$-AThe energy, carbohydrate, protein, fat and $\beta$ alaninelanine reported mild paraesthesia, which is a symptom commonly described when a single dose of 1,600 mg is ingested (Harris et al. 2006; Décombaz et al. 2012). The same symptoms are not observed when $1,600 \mathrm{mg}$ of $\beta$-alanine is provided in controlled release tablets (Saunders et al. 2012; Décombaz et al. 2012), suggesting that the strategy adopted in our study to slow the absorption of $\beta$-alanine (i.e., the addition of carboxymethyl cellulose added to the gelatin capsules) was not totally successful in preventing paraesthesia. Despite this, our data show that the participants as a group were not able to correctly guess the substance that they were ingesting, indicating that the blinding of the present study remained intact.

It can be concluded that 4 weeks of $\beta$-alanine supplementation improved repeated high-intensity cycling performance in both trained and non-trained participants. The findings of the present study indicate the efficacy of $\beta$ alanine as an ergogenic aid for high-intensity intermittent exercise regardless of the training status, highlighting that 
highly trained athletes can benefit from the use of $\beta$ alanine.

Acknowledgments The authors are grateful to all athletes and nonathletes who participate in the study and to Luana Farias de Oliveira for her technical assistance. Vitor de Salles Painelli was supported by the Conselho Nacional de Desenvolvimento Científico e Tecnológico (Grant \#130601/2011-0) and Fundação de Amparo à Pesquisa do Estado de Sao Paulo FAPESP (Grant 2013/04806-0). The authors are grateful to FAPESP (Grant 2010/11221-0). Roger C Harris is a UK old-age pensioner and an independent consultant to Natural Alternatives International.

Conflict of interest The authors declare that they have no conflict of interest.

Open Access This article is distributed under the terms of the Creative Commons Attribution License which permits any use, distribution, and reproduction in any medium, provided the original author(s) and the source are credited.

\section{References}

Artioli GG, Gualano B, Coelho DF, Benatti FB, Gailey AW, Lancha AH Jr (2007) Does sodium-bicarbonate ingestion improve simulated judo performance? Int J Sport Nutr Exerc Metab 17:206-217

Baguet A, Bourgois J, Vanhee L, Achten E, Derave W (2010) Important role of muscle carnosine in rowing performance. J Appl Physiol 109:1096-1101

Bate-Smith EC (1938) The buffering of muscle in rigour: protein, phosphate and carnosine. J Physiol 92:336-343

Belfry GR, Raymer GH, Marsh GD, Paterson DH, Thompson RT, Thomas SG (2012) Muscle metabolic status and acid-base balance during 10-s work: 5-s recovery intermittent and continuous exercise. J Appl Physiol 113:410-417

Bellinger PM (2014) $\beta$-Alanine supplementation for athletic performance: an update. J Strength Cond Res (in press)

Bellinger PM, Howe ST, Shing CM, Fell JW (2012) The effect of combined $\beta$-Alanine and $\mathrm{NaHCO}_{3}$ supplementation on cycling performance. Med Sci Sports Exerc 44:1545-1551

Bex T, Chung W, Baguet A, Stegen S, Stautemas J, Achten E, Derave W (2014) Muscle carnosine loading by $\beta$-Alanine supplementation is more pronounced in trained vs. untrained muscles. J Appl Physiol 116:204-209

Bishop D, Edge J, Goodman C (2004) Muscle buffer capacity and aerobic fitness are associated with repeated-sprint ability in women. Eur J Appl Physiol 92:540-547

Bogdanis GC, Nevill ME, Lakomy HK, Boobis LH (1998) Power output and muscle metabolism during and following recovery from 10 and $20 \mathrm{~s}$ of maximal sprint exercise in humans. Acta Physiol Scand 163:261-272

Boldyrev AA, Koldobski A, Kurella E, Maltseva V, Stvolinski S (1993) Natural histidine-containing dipeptide carnosine as a potent hydrophilic antioxidant with membrane stabilizing function. A biomedical aspect. Mol Chem Neuropathol 19:185-192

Chung W, Shaw G, Anderson ME, Pyne DB, Saunders PU, Bishop DJ, Burke LM (2012) Effect of 10 week $\beta$-Alanine supplementation on competition and training performance in elite swimmers. Nutrients 4:1441-1453

De Salles Painelli V, Roschel H, de Jesus F, Sale C, Harris RC, Solis MY, Benatti FB, Gualano B, Lancha AH Jr, Artioli GG (2013) The ergogenic effect of $\beta$-Alanine combined with sodium bicarbonate on high-intensity swimming performance. Appl Physiol Nutr Metab 38:525-532

De Salles Painelli V, Da Silva RP, de Oliveira Jr OM, de Oliveira LF, Benatti FB, Rabelo T, Guilherme JP, Lancha Jr AH, Artioli GG (2014) The effects of two different doses of calcium lactate on blood $\mathrm{pH}$, bicarbonate and repeated high-intensity exercise performance. Int J Sport Nutr Exerc Metab (in press)

Décombaz J, Beaumont M, Vuichoud J, Bouisset F, Stellingwerff T (2012) Effect of slow-release $\beta$-Alanine tablets on absorption kinetics and paresthesia. Amino Acids 43:67-76

Derave W, Ozdemir MS, Harris RC, Pottier A, Reyngoudt H, Koppo K, Wise JA, Achten E (2007) $\beta$-Alanine supplementation augments muscle carnosine content and attenuates fatigue during repeated isokinetic contraction bouts in trained sprinters. J Appl Physiol 103:1736-1743

Ducker KJ, Dawson B, Wallman KE (2013) Effect of $\beta$-Alanine supplementation on 2000-m rowing-ergometer performance. Int J Sport Nutr Exerc Metab 23:336-343

Dutka TL, Lamboley CR, McKenna MJ, Murphy RM, Lamb GD (2012) Effects of carnosine on contractile apparatus $\mathrm{Ca}^{2+}$ sensitivity and sarcoplasmic reticulum $\mathrm{Ca}^{2+}$ release in human skeletal muscle fibers. J Appl Physiol 112:728-736

Edge J, Bishop D, Goodman C (2006a) The effects of training intensity on muscle buffer capacity in females. Eur J Appl Physiol 96:97-105

Edge J, Bishop D, Goodman C (2006b) Effects of chronic $\mathrm{NaHCO}_{3}$ ingestion during interval training on changes to muscle buffer capacity, metabolism, and short-term endurance performance. J Appl Physiol 101:918-925

Harris RC, Tallon MJ, Dunnett M, Boobis L, Coakley J, Kim HJ, Fallowfield JL, Hill CA, Sale C, Wise JA (2006) The absorption of orally supplied $\beta$-Alanine and its effect on muscle carnosine synthesis in human vastus lateralis. Amino Acids 30:279-289

Hermansen L, Osnes JB (1972) Blood and muscle pH after maximal exercise in man. J Appl Physiol 32:304-308

Hipkiss AR, Michaelis J, Syrris P (1995) Non-enzymatic glycosylation of the dipeptide L-carnosine, a potential anti-protein-crosslinking agent. FEBS Lett 371:81-85

Hobson RM, Saunders B, Ball G, Harris RC, Sale C (2012) Effects of $\beta$-Alanine supplementation on exercise performance: a metaanalysis. Amino Acids 43:25-37

Hobson RM, Harris RC, Martin D, Smith P, Macklin B, Gualano B, Sale C (2013) Effect of $\beta$-Alanine with and without sodium bicarbonate on 2,000-m rowing performance. Int J Sport Nutr Exerc Metab 23:480-487

Howe ST, Bellinger PM, Driller MW, Shing CM, Fell JW (2014) the effect of $\beta$-Alanine supplementation on isokinetic force and cycling performance in highly-trained cyclists. Int J Sport Nutr Exerc Metab 23:562-570

Inbar O, Bar-Or O (1986) Anaerobic characteristics in male children and adolescents. Med Sci Sports Exerc 18:264-269

Jones G, Smith M, Harris R (2011) Imidazole dipeptide content of dietary sources commonly consumed within the British diet. Proc Nutr Soc 70:E363

Kendrick IP, Kim HJ, Harris RC, Kim CK, Dang VH, Lam TQ, Bui TT, Wise JA (2009) The effect of 4 weeks $\beta$-Alanine supplementation and isokinetic training on carnosine concentrations in type I and II human skeletal muscle fibres. Eur J Appl Physiol 106:131-138

Sale C, Artioli GG, Gualano B, Saunders B, Hobson RM, Harris RC (2013) Carnosine: from exercise performance to health. Amino Acids 44:1477-1491

Saunders B, Sale C, Harris RC, Sunderland C (2012) Effect of $\beta$ Alanine supplementation on repeated sprint performance during the Loughborough intermittent shuttle test. Amino Acids 43:39-47 
Stellingwerff T, Anwander H, Egger A, Buehler T, Kreis R, Decombaz J, Boesch C (2012) Effect of two $\beta$-Alanine dosing protocols on muscle carnosine synthesis and washout. Amino Acids 42:2461-2472

Tobias G, Benatti FB, de Salles Painelli V, Roschel H, Gualano B, Sale C, Harris RC, Lancha AH Jr, Artioli GG (2013) Additive effects of $\beta$-Alanine and sodium bicarbonate on upper-body intermittent performance. Amino Acids 45:309-317
Van Thienen R, Van Proeyen K, Vanden Eynde B, Puype J, Lefere T, Hespel P (2009) $\beta$-Alanine improves sprint performance in endurance cycling. Med Sci Sports Exerc 41:898-903

Weston AR, Myburgh KH, Lindsay FH, Dennis SC, Noakes TD, Hawley JA (1997) Skeletal muscle buffering capacity and endurance performance after high-intensity interval training by well-trained cyclists. Eur J Appl Physiol Occup Physiol 75:7-13 